\title{
Simulation Study of Energetic Ion Transport due to Alfvén Eigenmodes in LHD Plasma
}

\author{
Yasushi TODO, Noriyoshi NAKAJIMA, Masaki OSAKABE, Satoshi YAMAMOTO ${ }^{1)}$ \\ and Donald A. SPONG ${ }^{2}$ \\ National Institute for Fusion Science,322-6 Oroshi-cho, Toki 509-5292, Japan \\ 1) Kyoto University Pioneering Research Unit for Next Generation, Gokasho, Uji 611-0011, Japan \\ 2) Oak Ridge National Laboratory, Oak Ridge, Tennessee 37831, USA
}

(Received 16 November 2007 / Accepted 15 January 2008)

\begin{abstract}
The creation of holes and clumps in an energetic ion energy spectrum associated with Alfvén eigenmodes was examined using the neutral particle analyzer (NPA) on the LHD shot \#47645. The difference in slowingdown times between the holes and clumps suggested that the energetic ions were transported over $10 \%$ of the plasma minor radius. The spatial profile and frequency of the Alfvén eigenmodes were analyzed with the AE3D code. The phase space structures of the energetic ions on the NPA line-of-sight were investigated with Poincaré plots, where an oscillating Alfvén eigenmode was employed for each plot. The phase space regions trapped by the Alfvén eigenmodes appeared as islands in the Poincaré plots. The radial width of the islands corresponded to the transport distance of the energetic ions. Since island width depends on Alfvén eigenmode amplitude, it was found that Alfvén eigenmodes with amplitude $\delta B_{\mathrm{r}} / B \sim 10^{-3}$ transported energetic ions over $10 \%$ of the minor radius.
\end{abstract}

(c) 2008 The Japan Society of Plasma Science and Nuclear Fusion Research

Keywords: energetic ion transport, Alfvén eigenmode, Poincaré plot, LHD, neutral particle analyzer

DOI: $10.1585 /$ pfr.3.S1074

\section{Introduction}

The creation of hole and clump pairs in an energetic ion energy spectra associated with Alfvén eigenmodes was examined using the neutral particle analyzer (NPA) on the LHD shot \#47645 [1]. The frequencies of the Alfvén eigenmodes were roughly 55 and $68 \mathrm{kHz}$, respectively. Both the Alfvén eigenmodes had a toroidal mode number $n=1$. The hole and clump pairs were created at an energy of approximately $150 \mathrm{keV}$. The difference in the slowing-down times between the holes and clumps suggested that the energetic ions were transported across $10 \%$ of the plasma minor radius.

In this paper, we try to find candidates for the Alfvén eigenmodes using the AE3D code [2]. We also investigate the Alfvén eigenmode amplitude that is consistent with the energetic-ion transport, as suggested by the NPA data. Poincaré plots were prepared to investigate the phase space structures of the energetic ions on the NPA line-of-sight. The phase space regions trapped by the Alfvén eigenmodes appear as islands in the Poincare plots. The radial width of the islands corresponds to the effective transport distance of the energetic ions. Since the island width depends on the Alfvén eigenmode amplitude, we can determine the amplitude that is consistent with the NPA observation.

\section{Analysis of Alfvén Eigenmodes}

The Alfvén eigenmodes with a toroidal mode number $n=1$ in the LHD shot \#47645 were analyzed with the AE3D code, which is based on the Galerkin approach using a combined Fourier mode (poloidal/toroidal angle) finite element (radial) representation in Boozer coordinates. An MHD equilibrium was constructed in the Boozer coordinates. The safety factor profile and the Alfvén continuous spectra with toroidal mode number $n=1$ are shown in Figs. 1 and 2, respectively. Two toroidal Alfvén eigenmodes (TAE modes) were found with the eigenfrequencies of 42.7 and $79.1 \mathrm{kHz}$. The eigen-frequencies are comparable to the Alfvén eigenmode frequencies observed in the experiment. The spatial profiles of the electrostatic potential are shown in Fig. 3. The frequencies are also shown in Fig. 2. The primary poloidal harmonics of the TAE modes are $m=1$ and $m=2$. The primary poloidal harmonics have the same sign for the TAE mode with a frequency of $42.7 \mathrm{kHz}$, while they have the opposite signs for the mode with a frequency of $79.1 \mathrm{kHz}$.

\section{Analysis of Energetic-ion Orbit}

Energetic-ion orbits were calculated with different starting points on the NPA line-of-sight. The orbits were calculated in an MHD equilibrium constructed with the HINT code [3]. The energetic-ion energy was $150 \mathrm{keV}$ at which hole and clump creation in energy spectrum was 


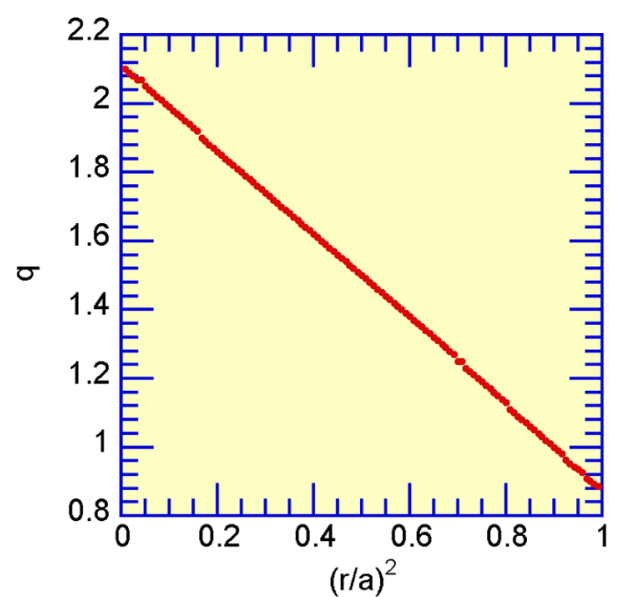

Fig. 1 Safety factor profile of the LHD shot \#47645.

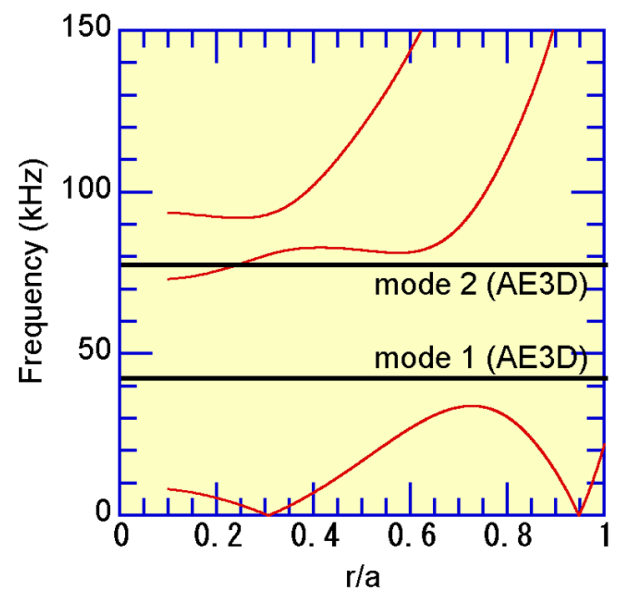

Fig. 2 Alfvén continuous spectra with toroidal mode number $n=1$ of the LHD shot \#47645. Black lines represent the frequencies of the toroidal Alfven eigenmodes analyzed with the AE3D code.

observed with the NPA. The pitch angle was determined by the direction of the NPA line-of-sight. A drift-kinetic description was employed for the energetic ions. The guiding-center velocity is given by

$$
\begin{aligned}
& \boldsymbol{u}=\boldsymbol{v}_{/ /}^{*}+\boldsymbol{v}_{E}+\boldsymbol{v}_{B}, \\
& \boldsymbol{v}_{/ /}^{*}=\frac{v_{/ /}}{B^{*}}\left[\boldsymbol{B}+\rho_{/ /} B \nabla \times \boldsymbol{b}\right], \\
& \boldsymbol{v}_{E}=\frac{1}{B^{*}}[\boldsymbol{E} \times \boldsymbol{b}], \\
& \boldsymbol{v}_{B}=\frac{1}{q_{\mathrm{h}} B^{*}}[-\mu \nabla B \times \boldsymbol{b}], \\
& \rho_{/ /}=\frac{m_{\mathrm{h}} v_{/ /}}{q_{\mathrm{h}} B}, \\
& \boldsymbol{b}=\boldsymbol{B} / B, \\
& B^{*}=B\left(1+\rho_{/ /} \boldsymbol{b} \cdot \nabla \times \boldsymbol{b}\right), \\
& m_{\mathrm{h}} v_{/ /} \frac{\mathrm{d} v_{/ /}}{\mathrm{d} t}=v_{/ /}^{*} \cdot\left[q_{\mathrm{h}} \boldsymbol{E}-\mu \nabla B\right],
\end{aligned}
$$

where $v_{/ /}$is the velocity parallel to the magnetic field, $\mu$ is the magnetic moment that is an adiabatic invariant, and $m_{\mathrm{h}}$
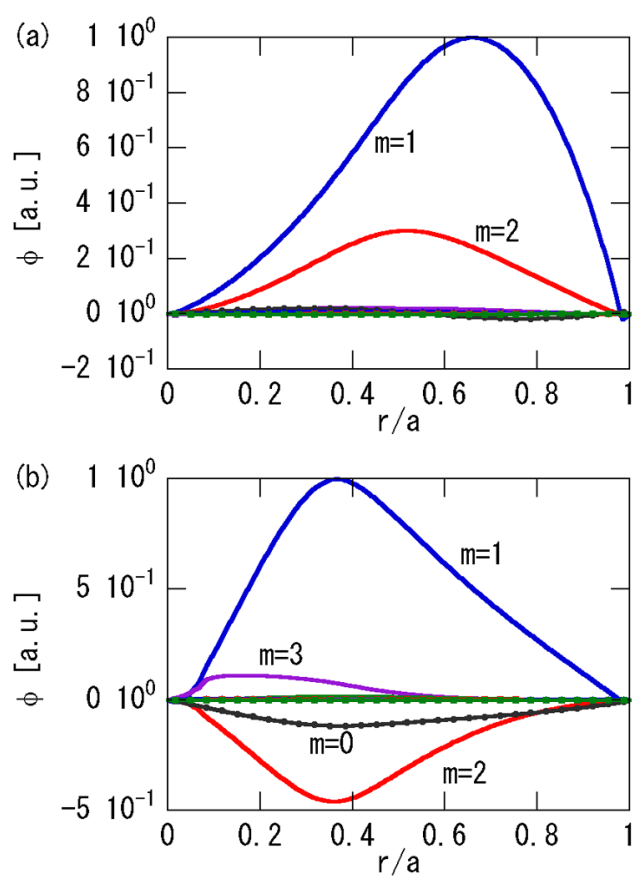

Fig. 3 Spatial profiles of the $n=1$ toroidal Alfven eigenmodes analyzed with the AE3D code for the LHD shot \#47645. The frequencies are (a) $42.7 \mathrm{kHz}$ and (b) $79.1 \mathrm{kHz}$, respectively.

and $q_{\mathrm{h}}$ are energetic ion mass and electric charge. In the MHD equilibrium used in the orbit calculation reported in this section, the electric field represented by $\boldsymbol{E}$ is zero.

The poloidal orbit frequency is defined by $\omega_{\theta}=$ $2 \pi / T_{\theta}$, where $T_{\theta}$ is the poloidal circulation time. The toroidal orbit frequency is defined by $\omega_{\varphi}=\Delta \varphi / T_{\theta}$, where $\Delta \varphi$ is the toroidal angle covered by the energetic ion during time $T_{\theta}$. In the experiment, the magnetic field was reversed so that the toroidal field is positive, $B_{\varphi}>0$, and the poloidal field is negative, $B_{\theta}<0$. The toroidal field is counter-clockwise when viewed from the top side. The energetic ions on the NPA line-of-sight moved in the direction of the magnetic field.

The orbit frequency of the energetic ions is defined by

$$
f_{m / n}=\left(n \omega_{\varphi}-m \omega_{\theta}\right) / 2 \pi
$$

where $n$ and $m$ are toroidal and poloidal mode numbers. We consider $n=1$ and $m=1$, which are identical to the primary poloidal harmonic of the TAE modes shown in Fig. 3. In Fig. 4, the orbit frequency is shown versus the major radius of the starting point on the NPA line-of-sight. We can see that energetic ions exist with the orbit frequencies 55 and $68 \mathrm{kHz}$, which are similar to the Alfvén eigenmode frequencies observed in the LHD experiment. These energetic ions resonate with the Alfvén eigenmodes. The major radius and pitch angle of the particles at the starting point on the NPA line-of-sight with the orbit frequencies of 55 and $68 \mathrm{kHz}$ are $\left(R=4.19 \mathrm{~m}, v_{/ /} / v_{\text {total }}=0.91\right)$ and $\left(R=4.10 \mathrm{~m}, v_{/ /} / v_{\text {total }}=0.89\right)$, respectively. 


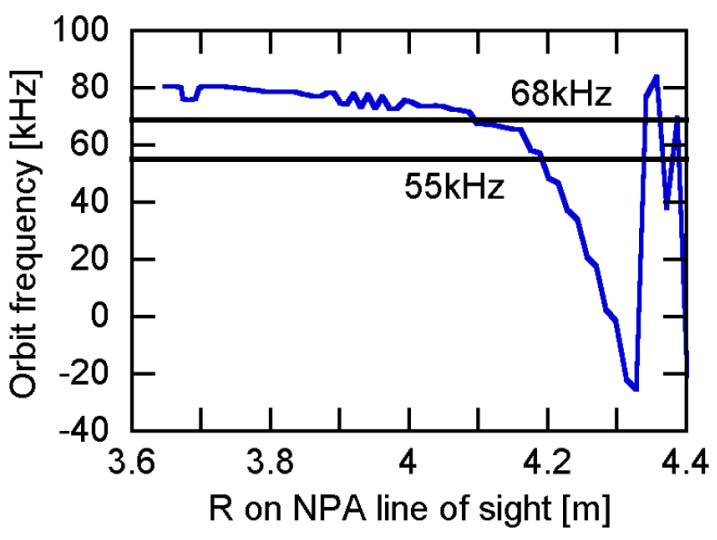

Fig. 4 Blue curve represents energetic-ion orbit frequency with $m / n=1 / 1$ versus major radius of the starting point on the NPA line-of-sight. Black lines denote Alfvén eigenmode frequencies observed in the LHD experiment.

\section{Phase Space Structure of Energetic Ions}

We now consider the distance the energetic ions are transported by the finite amplitude eigenmodes. To study this, we investigate Poincaré plots where only one eigenmode is taken into consideration and the amplitude of the eigenmode is at a constant value. We need a conserved variable to make Poincaré plots interpretable. In axisymmetric equilibria, we can find a conserved variable $E^{\prime} \equiv E-\omega P_{\varphi} / n$ in the interaction of a constant amplitude wave with frequency $\omega$ and toroidal mode number $n$. Here, $E$ is particle energy and $P_{\varphi}$ is canonical toroidal momentum. We choose particles that have a constant value of $E^{\prime}$. Then, with a single mode, we can obtain a conserved variable and make Poincaré plots that are easily interpretable. The phase space regions trapped by the eigenmodes appear as islands in the Poincaré plots. Since the island width depends on the Alfvén eigenmode amplitude, we can determine the amplitude that is consistent with the energetic ion transport. On the other hand, in nonaxisymmetric equilibria, we cannot find such a conserved variable. Then, we investigate an axisymmetric equilibrium comparable to the LHD equilibrium. Nevertheless, we would like to emphasize that this is the first attempt to understand the phase space structures in LHD plasmas with finite amplitude waves.

We investigate an axisymmetric equilibrium comparable to the LHD plasma \#47645. The parameters of the axisymmetric equilibrium are major radius $R_{0}=3.67 \mathrm{~m}$, minor radius $a=0.54 \mathrm{~m}$, toroidal magnetic field $0.5 \mathrm{~T}$, and safety factor profile $q(r)=2.11-1.23(r / a)^{2}$. This safety factor profile is a good approximation for that of the LHD plasma shown in Fig. 1. The value of $E^{\prime}$ is defined on the outer edge mid-plane at $\left(R-R_{0}\right) / a=0.7$ with energy $150 \mathrm{keV}$ and pitch angle $v_{/ /} / v_{\text {total }}=1$. As the harmonics of the two TAE modes other than $n=1$ are negligibly small, the major four poloidal harmonics
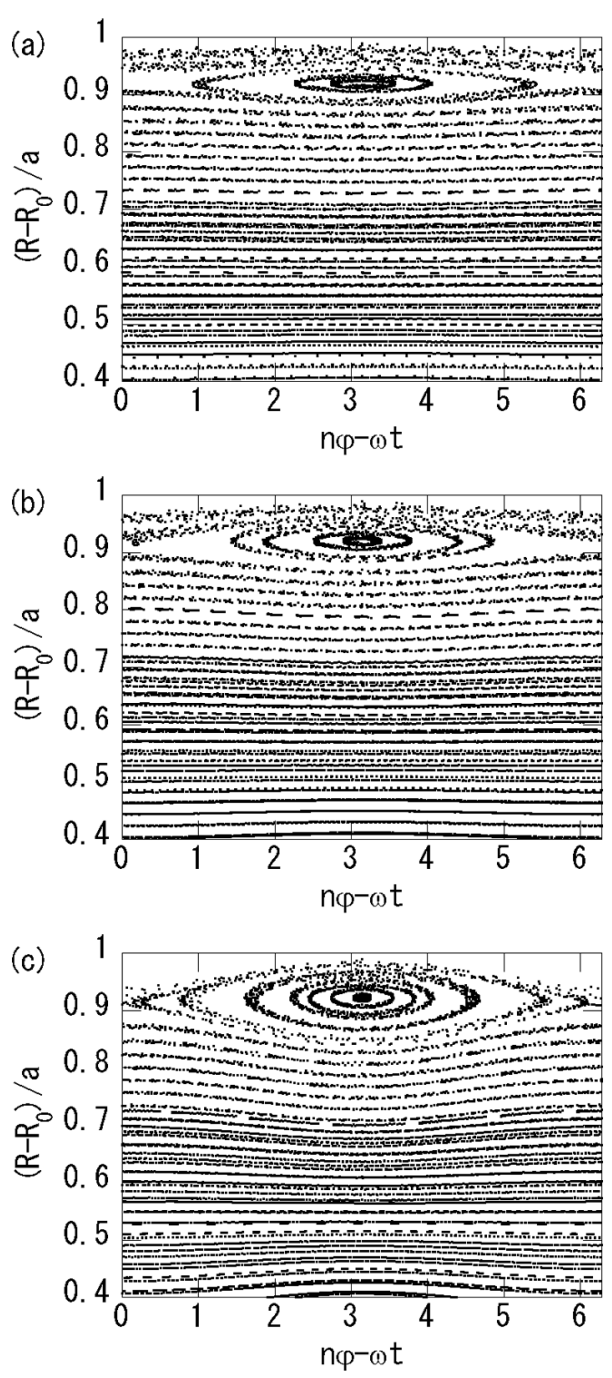

Fig. 5 Poincaré plots for the lower frequency TAE with amplitude (a) $\delta B_{\mathrm{r}} / B=10^{-3}$, and (b) $\delta B_{\mathrm{r}} / B=2 \times 10^{-3}$, and (c) $\delta B_{\mathrm{r}} / B=5 \times 10^{-3}$.

with $n=1$ were mapped to the axisymmetric equilibrium. The calculation of the electromagnetic field of the Alfvén eigenmodes from their potential harmonics is illustrated in Ref. [4]. The frequencies are renormalized to the experimental values, $55 \mathrm{kHz}$ for the lower frequency TAE shown in Fig. 3 (a) and $68 \mathrm{kHz}$ for the higher frequency TAE shown in Fig. 3 (b). The energetic-particle orbits were calculated in the electromagnetic field as the sum of the equilibrium magnetic field and the electromagnetic field of the Alfvén eigenmodes. In the Poincaré plot, we consider the major radius $\left(R-R_{0}\right) / a$ and phase, $n \varphi-\omega t$, of a particle each time the poloidal angle of the particle reaches the value of $\theta=0^{\circ}$.

The Poincaré plots for the lower frequency TAE are shown in Fig. 5. The phase space regions trapped by the Alfvén eigenmodes appear as islands in the Poincaré plots. The radial width of the islands corresponds to the transport distance of the energetic ions. The island width depends on 

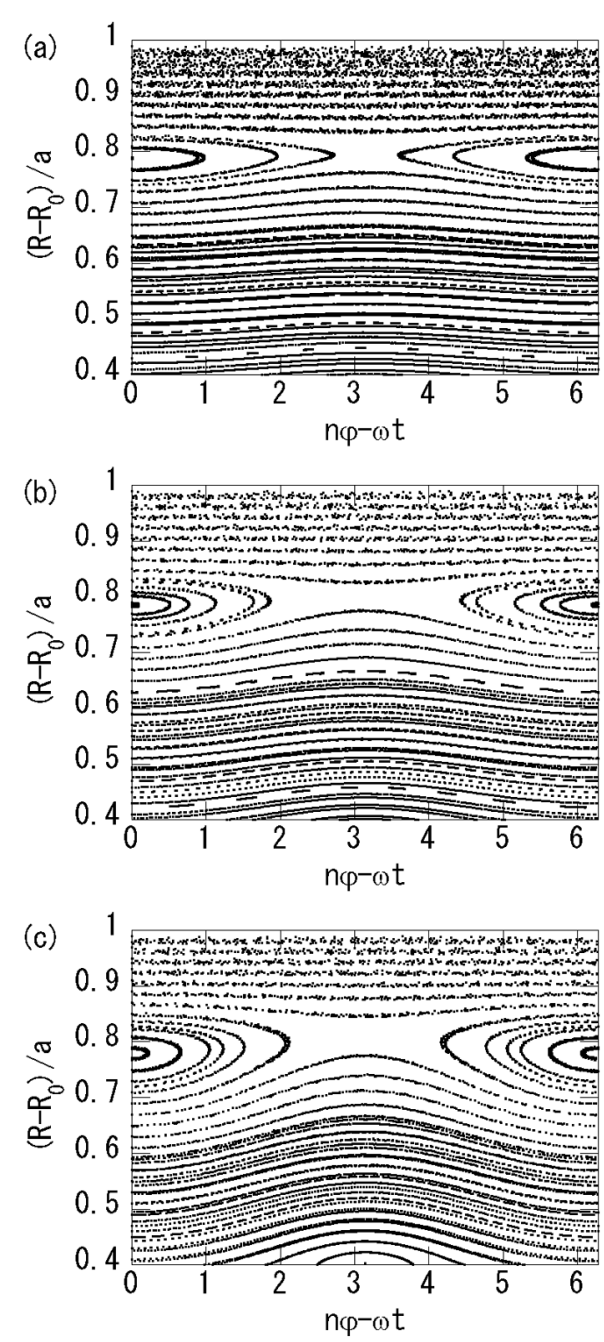

Fig. 6 Poincaré plots for the higher frequency TAE with amplitude (a) $\delta B_{\mathrm{r}} / B=5 \times 10^{-4}$, (b) $\delta B_{\mathrm{r}} / B=10^{-3}$, and (c) $\delta B_{\mathrm{r}} / B=2 \times 10^{-3}$.

the Alfvén eigenmode amplitude. For the lower frequency TAE, the amplitude $\delta B_{\mathrm{r}} / B=2 \times 10^{-3}$ transports energetic ions over $10 \%$ of the minor radius. The phase space structures for the higher frequency TAE in Fig. 6 are analyzed. Comparing Figs. 6(a), (b), and (c), we infer that the am- plitude of $\delta B_{\mathrm{r}} / B=10^{-3}$ is consistent with the transport of energetic ions over $10 \%$ of the minor radius.

\section{Summary}

The spatial profile and frequency of Alfvén eigenmodes have been examined using the AE3D code for the LHD shot \#47645, where the creation of hole/clump pairs in the energetic ion energy spectrum associated with the Alfvén eigenmodes was observed. The difference in slowing-down times between the holes and clumps observed in the experiment suggests that the energetic ions experienced rapid transport over $10 \%$ of the plasma minor radius. The phase space structures of the energetic ions on the NPA line-of-sight were investigated with Poincaré plots, where an oscillating Alfvén eigenmode is employed for each plot. The phase space regions trapped by the Alfvén eigenmodes appear as islands in the Poincaré plots. The radial width of the islands corresponds to the transport distance of the energetic ions. Since the island width depends on the Alfvén eigenmode amplitude, the Alfvén eigenmodes with amplitude $\delta B_{\mathrm{r}} / B \sim 10^{-3}$ resulted in island widths at about $10 \%$ of the minor radius, which is consistent with the experimental observations.

\section{Acknowledgements}

Numerical computations of the energetic-particles were performed at the Plasma Simulator (NEC SX7/160M5) of National Institute for Fusion Science. This work was supported by the NIFS Collaborative Research Program (NIFS06KTAT025 and NIFS06KDAT014).

[1] M. Osakabe et al., Nucl. Fusion 46, S911 (2006).

[2] D.A. Spong and Y. Todo, "Shear Alfvén spectrum and mode structures for $3 D$ configurations," 49th Annual Meeting of the Division of Plasma Physics (12-16 November 2007, Orlando), PP8.00098.

[3] K. Harafuji, T. Hayashi and T. Sato, J. Comput. Phys. 81, 169 (1989)

[4] Y. Todo, H.L. Berk and B.N. Breizman, Phys. Plasmas 10, 2888 (2003). 